\title{
Editorial
}

\section{Large-Scale Dynamics, Anomalous Flows, and Teleconnections 2015}

\author{
Anthony R. Lupo, ${ }^{1}$ Stephen J. Colucci, ${ }^{2}$ Igor I. Mokhov, ${ }^{3}$ and Yafei Wang ${ }^{4}$ \\ ${ }^{1}$ Department of Soil, Environmental, and Atmospheric Sciences, University of Missouri, \\ 302E Anheuser Busch Natural Resources Building, Columbia, MO 65211, USA \\ ${ }^{2}$ Department of Earth and Atmospheric Sciences, Cornell University, Bradfield Hall, Room 1116, Ithaca, NY 14853, USA \\ ${ }^{3}$ A.M. Obukhov Institute of Atmospheric Physics, Russian Academy of Sciences, 3 Pyzhevsky Pereulok, Moscow 119017, Russia \\ ${ }^{4}$ State Key Laboratory of Severe Weather, Chinese Academy of Meteorological Sciences, Beijing 100081, China
}

Correspondence should be addressed to Anthony R. Lupo; lupoa@missouri.edu

Received 5 August 2015; Accepted 5 August 2015

Copyright (C) 2016 Anthony R. Lupo et al. This is an open access article distributed under the Creative Commons Attribution License, which permits unrestricted use, distribution, and reproduction in any medium, provided the original work is properly cited.

The first version of this special issue was very successful, attracting ten peer-reviewed articles. The edition featured articles that had examined recent extreme events such as the Russian Drought of 2010, as well as issues of great interest to the LDAT international community such as the influence of interannual variability and climate change on LDAT. Since the first LDAT issue, some studies, for example, [1], examined the cold North American winter of 2013-2014 and suggested that a change in flow regime consistent with theory may be at least partly responsible for the occurrence of recent extremes in weather and climate. Also, in the last few years, there has been a warm sea surface temperature (SST) anomaly in the east Pacific and Gulf of Alaska region. This feature may be partly responsible for the anomalous prolonged ridging over the western part of North America that has led to dry conditions there [2].

In this issue, there is a diverse array of topics explored. One article examines the dynamics of an extremely strong and persistent Pacific Region blocking event in Jan-Feb 2014 (Figure 1(a)). Its author demonstrates that blocking events can survive flow regime transition as long as they are undergoing intensification at the time of the transition. Previous research suggested that blocking events did not survive flow regime transitions; see, for example, [3] and references therein. This article uses an enstrophy-based diagnostic shown to be effective in identifying flow regime transitions and published in the first LDAT issue. This pattern recurred during the winter of 2014-2015 (Figure 1(b)).
Another study demonstrates the ability of a three-layer quasi-geostrophic model to capture the basic essence of flow regimes associated with interannual and interdecadal variability in the Northern Hemisphere, including the Arctic Oscillation, North Atlantic Oscillation, and the Pacific North American teleconnections. In particular, they demonstrate the robustness of the positive phase in the Arctic Oscillation and the associated spatial anomalies. The idea of two or more basins of attraction representing different flow regimes in the large-scale distribution of the Northern Hemisphere height field has also had a long history of study in the LDAT community (e.g., $[4,5]$ and references therein).

The $Q$-vector is a well-known form of the $\omega$-equation, a diagnostic equation frequently used in weather analysis. This equation amalgamates the vorticity and thermal transports into one forcing process related to the divergence of the $Q$-vector. A paper in this issue adds latent heat release to the $Q$-vector formulation mathematically rather than isolating it as a separate forcing term. The article calls this an "extended" $Q$-vector. Then the authors demonstrate in a case of extratropical cyclone development that the extended Qvector better represents precipitation patterns that developed within the storm than the traditional Q-vector.

One research group submitted two studies on the interannual variability of storm tracks, one using a model to examine the role of the jet stream and associated cyclones played in the North American drought of 1988 and the flood of 1993. The authors found that the drought of 1988 was associated 


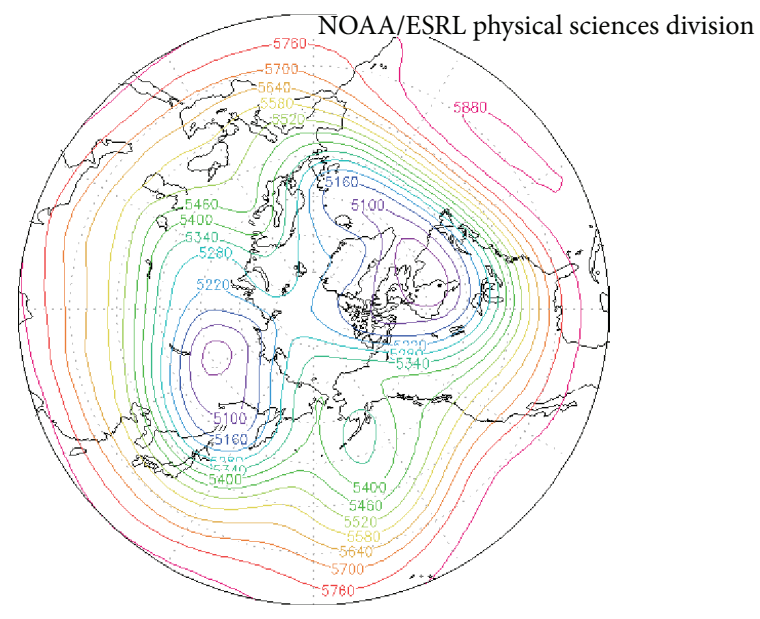

(a)

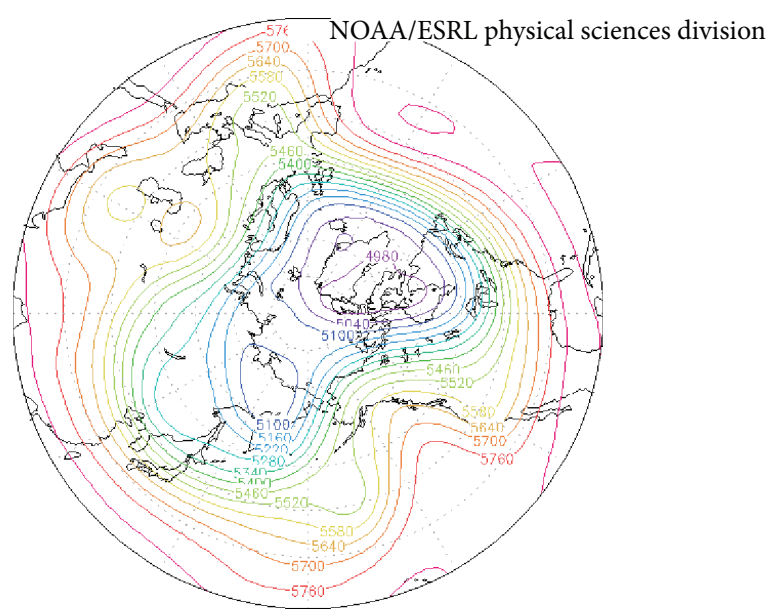

(b)

Figure 1: The mean $500 \mathrm{hPa}$ height field for (a) 23 Jan to 16 Feb 2014 and (b) 21 Feb to 2 Mar 2015, showing two blocking events that dominated their respective winter seasons in the Northern Hemisphere over the East Pacific. The time period encompasses the lifetime of the Pacific Region events, and contour intervals are at a $60 \mathrm{~m}$ interval. These were derived using reanalysis data found at http://www.esrl.noaa.gov/psd/data/reanalysis/reanalysis.shtml.

with strong ridging over North America (e.g., $[6,7]$ ), with a jet maximum over eastern Canada and another jet over southwest USA. The authors postulate that the proximity of the convergent regions of the jet maxima was responsible for setting the stage for ridging and that this may be a typical situation for the onset of La Niña. However, the 1993 flood was the result of Pacific moisture being brought into the Central United States by a jet stream lying southwest to northeast into North America. This situation was also associated with enhanced low-level flow out of the Gulf of Mexico, a situation more typical for El Niño years [8].

The above study demonstrates the importance of storm tracks in the large-scale atmospheric circulation, and variations in their location can have strong implications on agricultural and economic communities. Another study used the National Center for Environmental Prediction (NCEP) Climate Forecast System (CFS) and compares the ability of this model to simulate the interannual variability of the Northern Hemisphere storm tracks by comparing to the NCEP reanalysis data set. The authors of this study then compared the results of this model to those of the European Center for Medium Weather Forecasting (ECMWF) ERA40 data. They found the CFS was able to reproduce some features of the interannual variability of Northern Hemisphere storm tracks such as that associated with El Niño.

There have been changes globally in the general circulation. Researchers here also examine changes in tropical features over the last 15 years such as the Hadley Circulation and the Intertropical Convergence Zone (ITCZ) near South America. They found the Hadley Circulation region of South America and the adjacent South Atlantic had expanded poleward over the period of study. They also found that the Hadley Circulation has become more intense in the region and the possibility of Atlantic Niño. Sea surface temperatures (SSTs) were implicated in playing a role in the changes in the temperature and wind fields of the region. The poleward expansion of the Hadley Cell is thought to be a signal of a warmer climate (e.g., [9]).

Another study of tropical region dynamics uses three Regional Climate Models (RCMs) in order to assess their ability in simulating the interannual (El Niño and Southern Oscillation-related) occurrence of precipitation events that are associated with the West African Monsoon. This study was part of the Coordinated Regional Climate Downscaling Experiment (CORDEX) in which the models were driven by the same initial and boundary conditions. The models all did well in simulating regional easterly jets (e.g., African Easterly Jet). The models also simulated long-term rainfall adequately, but each model did a relatively poor job in capturing $850 \mathrm{hPa}$ features.

Finally, one group discusses the increases in surface turbulent (latent and sensible) heat flues over the Mediterranean Region that have occurred as the result of large-scale circulation changes over the region during the last half of the 20th century and at beginning of the 21st century. They examined the teleconnectivity of the Northern Hemisphere beyond the study region in order to determine what features were responsible for local climatic change. Using the temperature and humidity fields they found that variations in the strength and location (weak/west versus strong/east) of the Azores High explained most of the local variability, while the North Atlantic Oscillation was Less of a factor in the observed climate change.

The goals of this special issue series as stated in the first edition are to promote a better understanding of the outstanding questions in the LDAT community such as forecasting the onset and termination of particular flow regimes and blocking. Progress in this area has been slow but steady, and the study in this edition adds to our understanding of the relative role of large and synoptic-scale processes. 
Another goal is to understand how interannual variability and climate change may impact well-known teleconnections or the occurrence of anomalous flows. These may be associated with the occurrence of extreme weather around the globe. Many of the studies in this issue address the topic from a regional perspective, although a couple of studies looked at global variability. The results of this type of studies (in addition to those in this issue) have shown a variety of sometimes contradictory results [9]. This means there are still unanswered questions in the area of LDAT needed to improve our knowledge base and the regional or global numerical models we use. Advances in our understanding of LDAT issues will better inform long range forecasting and potential climate change scenarios and thus result in improved service to society.

\title{
Acknowledgments
}

The guest editors of this special issue in this journal would like to thank all the authors and reviewers who contributed their time and talent to our second edition.

\author{
Anthony R. Lupo \\ Stephen J. Colucci \\ Igor I. Mokhov \\ Yafei Wang
}

\section{References}

[1] J. A. Francis and S. J. Vavrus, "Evidence linking Arctic amplification to extreme weather in mid-latitudes," Geophysical Research Letters, vol. 39, Article ID L06801, 2012.

[2] S.-Y. Wang, L. Hipps, R. R. Gillies, and J.-H. Yoon, "Probable causes of the abnormal ridge accompanying the 2013-2014 California drought: ENSO precursor and anthropogenic warming footprint," Geophysical Research Letters, vol. 41, no. 9, pp. 3220 3226, 2014.

[3] A. R. Lupo, "A diagnosis of two blocking events that occurred simultaneously in the mid-latitude Northern Hemisphere," Monthly Weather Review, vol. 125, no. 8, pp. 1801-1823, 1997.

[4] K. C. Mo and M. Ghil, "Cluster analysis of multiple planetary flow regimes," Journal of Geophysical Research, vol. 93, no. 9, pp. 10927-10952, 1988.

[5] P.-A. Michelangeli, R. Vautard, and B. Legras, "Weather regimes: recurrence and quasi stationarity," Journal of the Atmospheric Sciences, vol. 52, no. 8, pp. 1237-1256, 1995.

[6] J. Namias, "Anatomy of great plains protracted heat waves (especially the 1980 U.S. summer drought)," Monthly Weather Review, vol. 110, no. 7, pp. 824-838, 1982.

[7] J. Namias, "Some causes of the United States drought," Journal of Climate and Applied Meteorology, vol. 22, no. 1, pp. 30-39, 1983.

[8] A. R. Lupo, A. R. N. B. Smith, P. E. Guinan, and M. D. Chesser, "The climatology of Missouri region dew points and the relationship to ENSO," National Weather Digest, vol. 36, no. 2, pp. 10-20, 2012.

[9] Intergovernmental Panel on Climate Change (IPCC), Climate Change 2013: The Physical Scientific Basis, Contributions of Working Group I to the Fifth Assessment Report of the Intergovernmental Panel on Climate Change, 2013, http://www.ipcc.ch. 

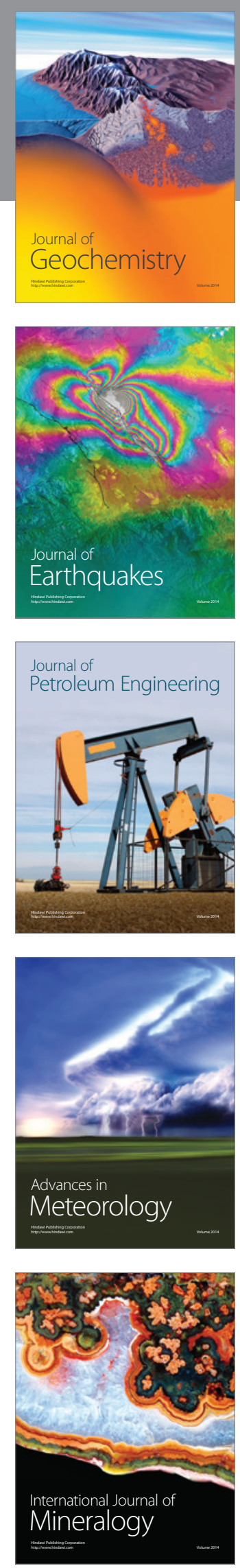
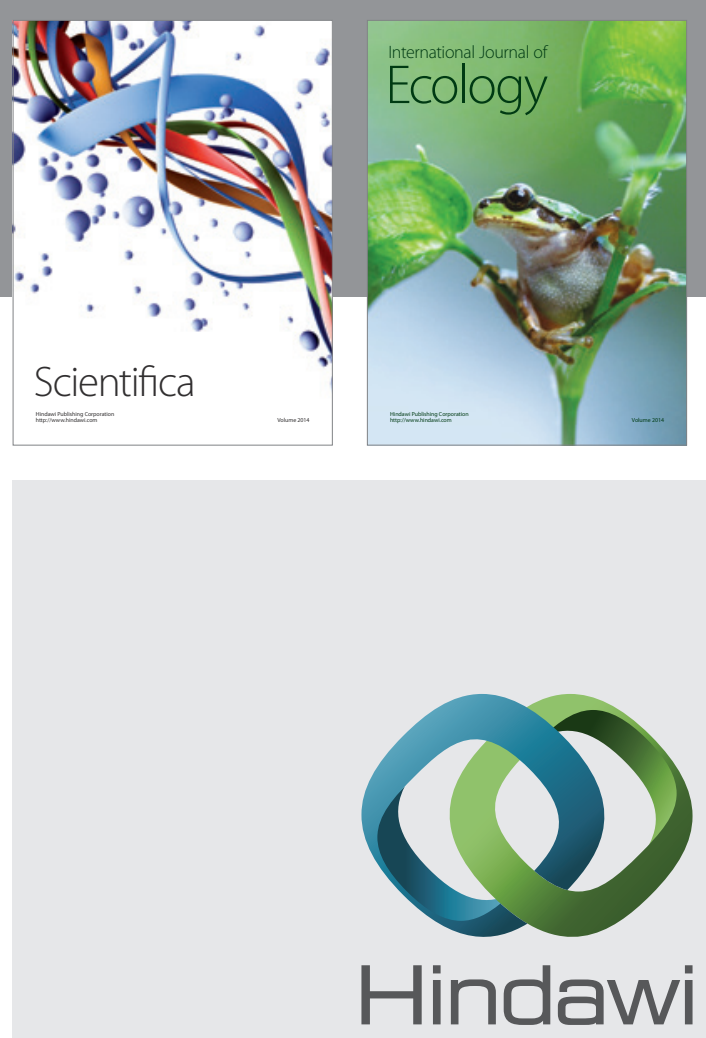

Submit your manuscripts at

http://www.hindawi.com
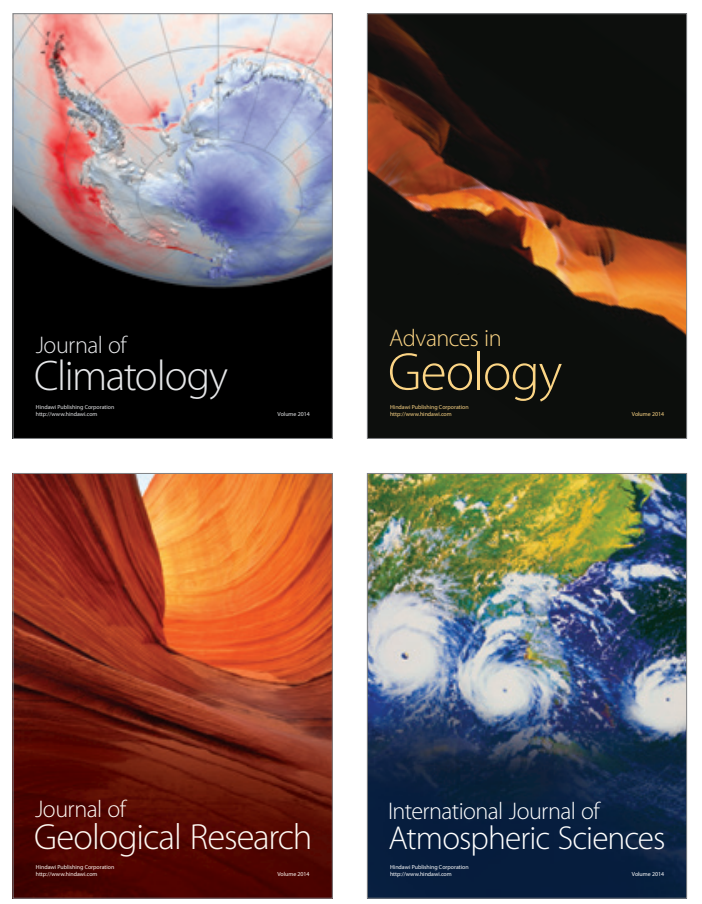

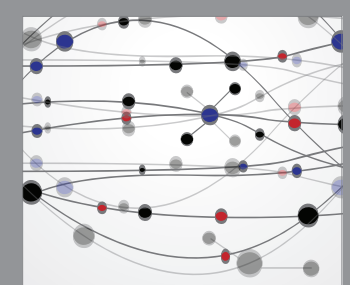

The Scientific

\section{World Journal}
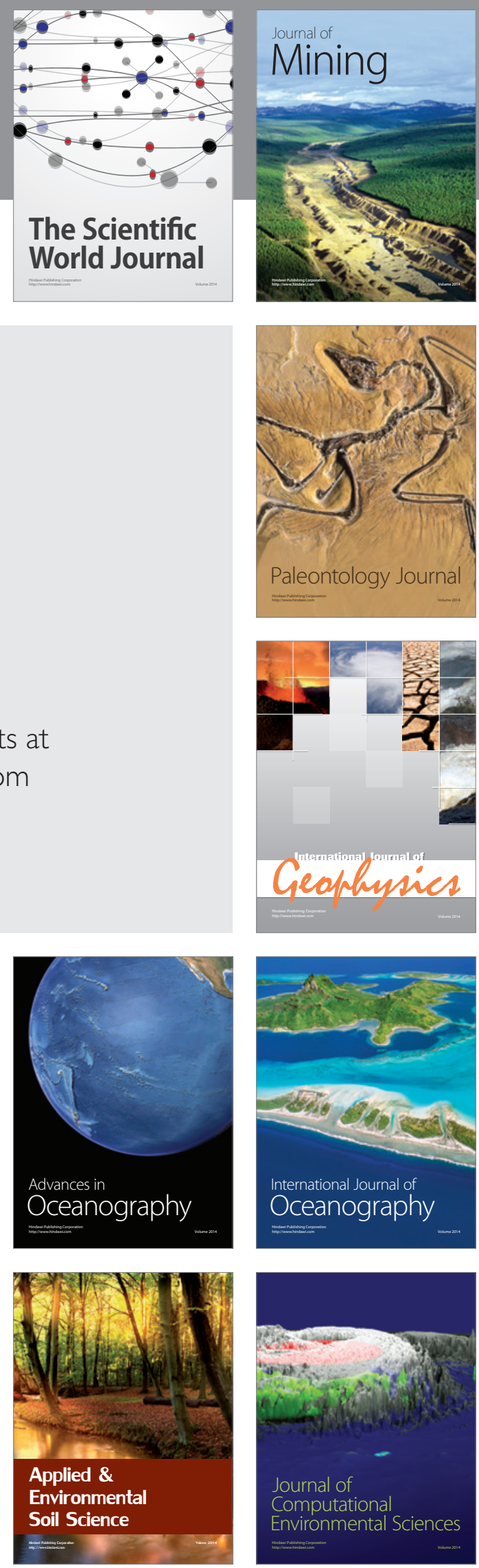